\title{
动物个性研究进展
}

\author{
李天韵, 刘依明, 王德利, 钟志伟* \\ 东北师范大学草地科学研究所,植被生态科学教育部重点实验室,吉林松嫩草地生态系统国家野外科学观测研究站, 长春 130024
}

\begin{abstract}
摘要: “个性”是指不同时空条件下动物种群个体间行为的稳定差异。大量的理论和实验性研究表明,个性差异在动物界普遍 存在, 其是种群多度和分布、物种共存及群落构建的重要驱动因子。介绍了动物个性的概念、分类及衡量指标, 将前人测量个性 类型的方法加以总结; 随后介绍了动物个性的生态学意义, 尤其是个性对动物生活史策略、种群分布与多度、群落结构和动态、 生态系统功能和过程以及疾病与信息传播等的影响。在此基础上,进一步分析了在人类活动增加等全球变化背景下,动物个性 如何调控动物个体行为、种群和群落动态对这些环境变化的响应。阐述了动物个性的形成与维持机制, 并对未来的研究方向进 行了展望。
\end{abstract}

关键词: 动物个性; 生活史策略; 分布与多度; 种间关系; 群落动态; 生态系统过程; 环境变化

\section{Concepts, research progresses and prospects of animal personality}

LI Tianyun, LIU Yiming, WANG Deli, ZHONG Zhiwei*

Institute of Grassland Science, Key Laboratory of Vegetation Ecology of the Ministry of Education, Jilin Songnen Grassland Ecosystem National Observation and Research Station, Northeast Normal University, Changchun 130024, China

\begin{abstract}
Interspecific trait variation has long been considered as a conceptual foundation for our understanding of species interactions and community structure. In particular, ecologists realize the important role that animal behavior plays in shaping ecological processes. Although most studies commonly supposed that the functional diversity occurs at the level of the species and ignored individual variation. Recently, an emerging field of interest in animal behavior, the studies of animal personalities focus on how individual differences in behavior, as well as behavioral correlations affect the fitness of individuals in various ecological contexts. "Animal personality" describes the common phenomenon that individual behavioral differences are consistent over time and across situations, which covers numerous traits, such as willingness to take risks, the reaction of an animal to novel environment, activity, aggressiveness, and sociality. The majority of theoretical and empirical studies suggest that personality differences are a widespread phenomenon throughout the animal kingdom, and it limits behavioral plasticity and explains non-optimal behavior, which has far-reaching implications for understanding the mechanism of species interactions and coexistence. In this article, we review the concepts, trait categories, and measurement methods of animal personality. We then aim to provide a comprehensive inventory of the potential consequences of personality differences for ecological and evolutionary patterns and processes, we discuss the ecological implications of personality, including life-history strategies, population distribution and abundance, species interactions, community structure and dynamics, ecosystem processes and disease and information transmission. In addition, a critical ecological and evolutionary issue is the need to better understand individual responses to human-induced rapid environmental change that often puts organisms in evolutionarily novel conditions. Behavior is predicted to be a major

基金项目:国家自然科学基金(32061143027,31770520); 中央高校基本科研业务费专项资金(2412020FZ019); 兰州大学草地农业生态系统国家 重点实验室开放课题( SKLGAE201904)

收稿日期: 2019-12-02; 网络出版日期:2021-08- 16

* 通讯作者 Corresponding author.E-mail: zhongzw822@ nenu.edu.cn
\end{abstract}


factor driving responses of individuals to global change because it largely determines how animals interact with the environment. This intraspecific trait variation differs strikingly in their ability to cope with responses to human activity and urbanization induced rapidly environmental change. Thus, incorporating animal personalities can provide great insight into our understanding of the ecological and evolutionary consequences of global change and help either aid declining species or better manage invasive or pest species. Moreover, we discuss the proximate and ultimate mechanisms underlying individual variation in personality traits, including the effects of ecological, genetic, physiological, and neuroendocrine factors on the maintenance of variation in personality traits in natural animal populations. The animal personality can form a key bridge in an interdisciplinary approach that integrates proximate mechanisms (genetics, development, and neuroendocrine bases) with ecology and evolution. Finally, the future research direction is prospected.

Key Words: animal personality; life history; distribution and abundance; species interactions; community dynamics; ecosystem processes; environmental change

“个性” 这一术语最早起源于人类心理学 ${ }^{[1]}$ 。随着研究的日益深人, 研究者发现人类之外的其他动物同 样具有个性, 并类比提出了“动物个性 ( animal personality)” 这一概念 ${ }^{[2]}$ 。一般认为, 动物个性是指动物具有 稳定的个体间差异的各类行为, 是动物对所在种群和环境的适应性结果 ${ }^{[3]}$ 。Réale 等 ${ }^{[4]}$ 提出个性与性格 (temperament) 和应对方式 (coping styles) 和行为综合症 (behavioral syndromes) 等可视为相同或相近概念, 并将 个性分为勇敢性 (boldness)、探索性 (exploration)、活跃性 (activity)、攻击性 (aggressiveness) 及社会性 ( sociability) 等 5 个维度。不同个性维度之间相互关联,组成了动物行为综合征 ${ }^{[5]}$ 。

动物个性涉及的研究对象相当广泛, 包括脊椎动物如哺乳类、鸟类、爬行类、两栖类和鱼类等, 以及无脊椎 动物如节肢动物等 ${ }^{[6-7]}$ 。过去, 这些动物个体间的行为差异通常被研究者当作观察过程中的 “噪音” 而未受 到重视 ${ }^{[8-9]}$ 。然而在最近 20 年中, 越来越多的理论与实验性研究表明, 动物个性反映了动物的生活史策略, 并与其物种形成、遗传变异及种群动态等紧密关联, 具有广泛的进化和生态学意义, 因而具有重要的理论研究 和应用价值 ${ }^{[10]}$ 。

动物的行为一直是生态学家关注的重点 ${ }^{[11-12]}$, 动物个性研究的兴起加深了人们对动物行为的理解, 改变 了行为生态学的传统观点 ${ }^{[13]}$ 。行为生态学家认为行为具有无限的可塑性, 即所有的个体在环境中都能表现 出最适宜的行为 ${ }^{[4]}$, 而个性概念本身意味着行为可塑性是有限的, 为一些个体在环境中表现出的非最适行为 作出合理解释 ${ }^{[14]}$ 。动物个性可以决定特定环境下个体的适合度。例如, 在草地生态系统中, 勇敢的蟋蟀个体 可以捕获更多猎物并有利于繁殖率的提高; 但当捕食者 (如鸟类) 出现时, 谨慎的个体则能更好地躲避危险, 从而产生更多的后代 ${ }^{[15]}$ 。在国际上, 个性在动物个体中的普遍性与重要性已经得到了广泛认可 ${ }^{8,16]}$, 但国内 对该领域的关注相对不足 ${ }^{[17-18]}$ 。本文将结合近年来动物个性的相关研究, 阐述动物个性的概念与维度, 系统 总结动物个性对物种共存、种群动态以及群落构建的影响与机制, 并对动物个性领域未来的研究方向予以 展望。

\section{1 个性的概念及测量方法}

\section{1 个性的概念}

有关动物个性的研究一直在深人, 涉及的物种日益增多, 因此关于动物个性的术语和定义也在不断发展 (表 1)。

\section{2 个性的分类及测量方法}

动物个性的分类及其定义如下 ${ }^{[4]}$ : (1) 勇敢性, 指个体对危险环境的反应。Wilson 等 ${ }^{[25]}$ 发现勇敢的蓝腮 太阳鱼 (Lepomis macrochirus) 能更快适应实验室环境, 捕获更多猎物。周杨等 ${ }^{[26]}$ 发现马稱 (Moschus sifanicus) 泌香量与非交配季节胆量之间存在显著负相关，而雌槄繁殖成效与交配季节胆量之间存在显著正相关。 
(2) 探索性,指个体对新环境的适应能力。在欧亚大山雀 (Parus major) 种群中,探索性策略的选择取决于竞 争的激烈程度, 竞争激烈时, 探索性强的个体具有明显优势; 竞争缓和对探索性弱的个体更有利 ${ }^{[27]}$ 。(3)活跃 性, 描述个体的活动能力, 其是将行为与摄食率、代谢消耗和捕食风险联系在一起的关键特征, 与种群及群落 动态息息相关 ${ }^{[28]}$ 。研究发现, 鲑鳟鱼类的标准代谢率 (standard metabolic rate, SMR) 与活跃性高度关联,例如 美国红点鲑 (Salvelinus fontinalis) 的 SMR 与其个体活跃程度呈正相关 ${ }^{[29]}$; 反过来, SMR 越高的欧鲈 (Dicentrarchus labrax) 个体的活跃程度也相对较高 ${ }^{[30]}$ 。(4)社会性, 指对同种个体的反应, 表现为集群型和离 群型。Cote 和 Clobert ${ }^{[31]}$ 将不同社会性的胎生蚚蜴 (Lacerta vivipara) 幼虫释放到不同密度的种群中, 以测试它 们的分散和定居行为, 发现个体的社会性不随时间而改变, 并且这种社会性决定其后来的栖息地选择与定居 模式,此研究表明社会性显著影响分布策略。(5) 攻击性,指攻击其它个体的倾向。Riechert 和 Hedrick ${ }^{[28]}$ 发 现北美漏斗网蜘蛛 (Agelenopsis aperta) 种群中, 攻击性强的个体对猎物和同类均表现出更明显的侵略性, 并且 对捕食者袭击的反应更迅速。具体的个性测量方法见表 2 。

\section{表 1 动物“个性”行为的术语与定义}

Table 1 The terms and definitions of animal personality

\begin{tabular}{|c|c|c|}
\hline $\begin{array}{l}\text { 术语 } \\
\text { Terms }\end{array}$ & $\begin{array}{l}\text { 定义 } \\
\text { Definition }\end{array}$ & $\begin{array}{c}\text { 文献来源 } \\
\text { Source }\end{array}$ \\
\hline \multirow[t]{4}{*}{ 个性 Personality } & (1)个体在情绪、思维和行为等方面表现出的稳定特征。 & {$[2]$} \\
\hline & (2) 动物个体与同一物种中同性同龄的其他个体之间稳定的行为差异。 & {$[19]$} \\
\hline & (3) 随时空变化,个体间行为的稳定性差异。 & {$[4]$} \\
\hline & $\begin{array}{l}\text { (4) 种群内行为倾向的系统性和结构性差异,这种倾向在一段时间内是稳定的,并且不同环境中这些行 } \\
\text { 为倾向相互关联。 }\end{array}$ & {$[10]$} \\
\hline \multirow[t]{4}{*}{ 性格 Temperament } & (1)个体所固有的,用于调节其活动性、反应性、情绪性、社会性表达的相对稳定的基本配置。 & {$[20]$} \\
\hline & (2) 个体在不同情况下情绪和行为方式的典型风格,这种风格在生命早期已经出现,但并非一成不变。 & {$[21]$} \\
\hline & (3) 人类的研究中,可遗传的、早期出现、贯穿个体一生的行为倾向,是个性的基础。 & {$[2]$} \\
\hline & (4) 由不同条件下神经系统决定的一种复杂的行为类型。 & {$[22]$} \\
\hline 行为综合征 & (1) 不同条件下一组相互关联行为的集合。 & {$[5]$} \\
\hline \multirow[t]{3}{*}{ Behavioral syndromes } & $\begin{array}{l}\text { (2) 行为综合征包括个体内和个体间的行为稳定性。个体内部的稳定性指个体在不同时空条件下一致 } \\
\text { 的行为方式, 即个体具有某种行为类型; 个体间的稳定性发生在不同行为类型的个体之间, 表现出个体 } \\
\text { 之间行为的相关性。 }\end{array}$ & {$[9]$} \\
\hline & $\begin{array}{l}\text { (3) 核心包括两个关键点:首先, 个体在行为上表现出个体内的稳定性, 意味着有限的行为可塑性; 其 } \\
\text { 次,行为与环境相互关联。 }\end{array}$ & {$[23]$} \\
\hline & (4)种群中的个体在两种或两种以上功能不同行为中表现出的一致性/相关性。 & {$[24]$} \\
\hline
\end{tabular}

表 2 动物个性的测量方法

Table 2 Summary of methodologies measuring personality

\begin{tabular}{|c|c|c|c|}
\hline $\begin{array}{l}\text { 个性指标 } \\
\text { Personality traits }\end{array}$ & $\begin{array}{l}\text { 测试方法 } \\
\text { Tests }\end{array}$ & $\begin{array}{l}\text { 观察指标 } \\
\text { Example of operational definition }\end{array}$ & $\begin{array}{c}\text { 文献来源 } \\
\text { Sources }\end{array}$ \\
\hline \multirow[t]{5}{*}{ 勇敢性 Boldness } & 危险刺激测试 & 对捕食者回避的反应时间 & {$[32]$} \\
\hline & & 对食物区附近的惊吓反应和返回食物区的时长 & {$[33]$} \\
\hline & & 进人陷阱后反应时长或被捕食概率 & {$[34]$} \\
\hline & 诱捕实验 & 进攻倾向及捕食成功率 & {$[35]$} \\
\hline & 干扰测试 & 再次移动的反应时长 & {$[36]$} \\
\hline \multirow[t]{2}{*}{ 探索性 Exploration } & 旷场实验 & 移动距离和速度、排泄时间、育幼时间 & {$[37]$} \\
\hline & 洞板实验 & 接近食物附近新物体的反应时间 & {$[38]$} \\
\hline 活跃性 Activity & 旷场实验/笼罩观察 & 移动距离 & {$[39]$} \\
\hline 社会性 Sociability & 分离测试 & 离开群体的反应/从孤立状态到加人群体的反应时间 & {$[40]$} \\
\hline 攻击性 Aggressiveness & 同种互作 & 对同种个体出现的反应/发起攻击的延迟时间 & {$[41]$} \\
\hline
\end{tabular}




\section{2 动物个性的生态学意义}

\section{1 生活史策略}

动物个性与个体的生活史策略密切相关。首先, 动物个性差异可以影响个体的存活率 ${ }^{[14]}$ 。例如, Richardson 等 ${ }^{[42]}$ 发现吸蜜鸟 (Notiomystis cincta) 的勇敢性与其幼年的存活率呈正相关, 而 Ballew 等 ${ }^{[43]}$ 对大口 黑鲈 (Micropterus salmoides) 的研究却得到相反结果。上述研究同时证实了动物个性对个体存活率的影响, 其 结果的差异可能是由捕食者捕食强度的不同所导致。其次, 动物个性可以驱动种群生长率-死亡率的权 衡 ${ }^{[44]}$ 。通常来说, 支持高生长率的个性特征同时也会增加死亡率。例如, 在迷彩箭毒蛙 (Dendrobates auratus) 的幼体中, 高度活跃的个体受食速度更快, 因此生长率更高, 但其也面临着更高的捕食或疾病风险 ${ }^{[45]}$ 。Biro 等 ${ }^{[46]}$ 在对虹䲡鱼 (Oncorhynchus mykiss) 的生活史研究中同样得到类似结论。此外,动物个性在配偶选择和生 殖方面也发挥重要作用 ${ }^{[47-48]}$ 。在欧亚大山雀种群中, 探索性相似的个体交配所抚育的后代健康状况更好 ${ }^{[49]}$ 并且繁殖率更高 ${ }^{[27]}$ 。Martin-Wintle 等 ${ }^{[50]}$ 发现大熊猫(Ailuropoda melanoleuca) 中某些个性的特定配对表现出 更好的繁殖性能, 如雄性比其配偶攻击性更强时, 两者更容易交配并生产后代; 易兴奋的雄性与冷静的雌性交 配会有更高的繁殖效率,这些结果暗示了基于个性特征的交配模式在濒危物种保育计划中潜在的重要性。

2.2 种群多度及分布

动物个性对种群的多度和分布有重要影响。因为个性特征决定了个体在行为模式、栖息地利用、食性选 择以及捕食策略的差异, 这种分化将决定种群的多度和分布 ${ }^{[51]}$ 。首先, 资源和生境利用的差异缓解了个体间 的竞争, 因此个体能够更有效并广泛地利用资源和生境, 从而促进了种群多度的增加 ${ }^{[52]}$ 。例如, 活跃程度高 的三刺鱼 (Gasterosteus aculeatus) 能够获得更好的资源, 但同时面临着更强的捕食风险, 资源利用与风险的权 衡最终决定了种群多度 ${ }^{[53]}$ 。其次,动物个性差异是个体在栖息地中呈现非随机分布的重要原因。例如,攻击 性强的西蓝鸲 (Sialia mexicana) 个体倾向于在食物资源丰富的区域建立领地,而攻击性弱的个体则只能栖息 在资源贫癐的区域 ${ }^{[54]}$ 。Sih 等 ${ }^{[55]}$ 发现河滨钝口螈 (Ambystoma barbouri) 种群中勇敢的个体在很小的、快要干 涸的池塘中占优势, 因为它们可以捕食足够多的猎物; 而谨慎的个体则适合在更大、食物更加充足的池塘中 生活。

\section{3 群落构建与生态系统过程}

生物群落是通过生物体与其环境或其他物种之间复杂的相互作用所形成的, 动物个性差异通过对物种互 作的影响, 会进一步反馈于群落结构及生态系统过程 ${ }^{[56]}$ 。捕食者的个性往往决定了其遇到和捕杀的猎物种 类, 进而影响猎物群落的组成和结构 ${ }^{[57]}$ 。Post 等 ${ }^{[58]}$ 在新英格兰海岸的湖泊中对状锯腹鲱 (Alosa pseudoharengus) 行为的研究发现, 由于个体生活史和采食行为的差异, 状锯腹鲱对不同种类浮游动物的捕食 程度也不同, 因此对后者群落物种组成和多样性的影响也存在差异。类似地, 在北美的淡水生态系统中, 水蚤 的竞争能力强于桡足动物, 而活跃的蜻蜓通常选择攻击水虫, 使得竞争力较弱的桡足动物得以生存和发展, 从 而改变了浮游动物群落组成 ${ }^{[59]}$ 。还有研究发现, 在孔雀鱼 (Poecilia reticulata) 种群中, 由于不同个性的个体 食性选择的差别, 造成了其对植物生产力、植物残体分解和养分循环等关键生态系统功能和过程影响的差 异 ${ }^{[60]}$ 。因此, 捕食者个性的差异, 可以通过影响捕食者-猎物-初级生产者之间关系的强弱,进而对整个生物群 落的组成和结构产生影响, 并最终反馈于生态系统的功能和过程。

\section{4 疾病与信息传播}

动物个性可以对传染病的流行、动态及其爆发强度产生强烈影响。首先, 个性差异造成了个体空间分布 不同, 因此暴露于不同的传染源 ${ }^{[25]}$ 。其次, 个性特征在社会网络的结构和动态上有很大不同,这是病毒传播 的一个关键性决定因素 ${ }^{[61]}$ 。例如, 虽然三刺鱼中勇敢个体间的相互作用通常比胆怯的个体间更少, 但是这种 互作更均匀, 涉及的群体成员更多, 从而潜在地促进了病毒的传播 ${ }^{[62]}$ 。最后, 个性会影响个体接触寄生虫或 者感染疾病的可能性。探索性低的个体患病率相对较低, 对疾病导致的灭绝具有更大的缓冲作用 ${ }^{[63]}$ 。例如, 
勇敢的西伯利亚花栗鼠 (Tamias sibiricus) 可以利用更大的栖息地,但也寄生更多的蜱虫 ${ }^{[64]}$ 。

个性还可以调控动物之间的信息传递。信息通过社会学习而传播,依赖于社会网络结构和获取信息的能 力,而这些能力又受个性特征的影响,如社会性、探索性、勇敢性和反应能力 ${ }^{[65]}$ 。此外,群体内行为类型的混 合对于集体决策 ${ }^{[66]}$ 和社会信息 ${ }^{[67]}$ 的使用至关重要,不同行为类型的个体通常在特定信息的获取和传播中起 着关键作用。Aplin 等 ${ }^{[68]}$ 研究学习技巧如何在种群中传播, 设计了欧亚大山雀的“谜箱实验”, 发现勇敢的个 体传播信息更快,因为它们接触的个体更多,因此个性会影响鸟类社交网络的信息传递。

\section{3 环境变化对动物个性的影响}

当前, 人类活动的增加和气候变化正严重威胁着动物的生存及其多样性的维系。个性在很大程度上决定 了动物个体行为、种群多度和分布对环境变化的响应 ${ }^{[69]}$ 。研究动物对环境变化的响应与其个性之间的关系, 有助于更好地理解全球变化背景下动物物种共存及其群落构建机制 ${ }^{[52]}$ 。

在环境变化条件下, 个性往往决定着动物个体对栖息地的选择 ${ }^{[70]}$ 。通常情况下,勇敢的个体能更好地在 城市栖息地中存活,而胆怯的个体则主要分布在未被开发的农村地区 ${ }^{[71]}$ 。Lapiedra ${ }^{\text {等 }}{ }^{[72]}$ 对沙氏变色蜥 (Anolis sagrei) 的研究支持了这一结论,他们发现城市的蚚蜴比附近森林中的蚚蜴抵御人类干扰的能力更强, 因为城市蚚蜴个体表现出更强的探索性。Sol 等 ${ }^{[73]}$ 对农村和城市鸟类行为的研究也得到类似结果。然而, Miranda 等 ${ }^{[74]}$ 通过对欧亚乌鸫 (Turdus merula) 个性的研究, 发现乌鸦个体对新事物有两种不同反应: 接近( 嗜 新性) 和躲避 (恐新性)。城市中的乌斩比农村的乌冻更惧怕新环境, 因为前者适应了城市相对稳定的资源与 气候环境 (如人类的喂食和适宜的温度), 而与新的、潜在的危险环境接触较少。因此, 当动物面临环境变化 时, 自然选择会倾向于某种个性, 个性差异通过产生与其生态环境相适应的行为, 促进了动物在新环境中的 生存。

\section{4 个性的形成与维持机制}

动物种群在进化过程中如何形成与维持个性一直是学者关注的重点。研究者分别从远因 (进化)、近因 (生理、发育等) 和外部环境变化等角度对动物个性的形成进行了阐释。首先, Wolf 等 ${ }^{[75]}$ 从进化的角度分析 了动物个性的形成, 发现当前和未来繁殖率之间的权衡会导致种内差异的出现, 具体表现为对未来期望较高 的个体更倾向于规避风险,这一结果从进化角度解释了动物后代不同个体行为的稳定性差异。程琪等 ${ }^{[76]}$ 对 高原鼠兔 (Ochotona curzoniae) 个性特征的地理变异的研究表明, 高原鼠兔个性特征中主要行为参数并无显著 的地理差异, 因而推测鼠兔个性可能受到遗传因素的影响要大于外部环境条件变化的影响。其次, Réale 等 ${ }^{[77]}$ 则认为动物个性主要受到激素和代谢等生理条件和过程的影响。也有研究表明, 动物许多个性的形成 和分化都受到神经内分泌通路的调控,包括下丘脑-垂体-肾上腺轴 (hypothalamic-pituitary-adrenal axis) 以及交 感和副交感神经系统 ${ }^{[78]}$ 。此外, 大脑前额皮质 (prefrontal cortex, PFC) 也与动物个性的形成高度相关, 动物的 攻击性及行为冲动很大程度受到 PFC 的调控 ${ }^{[79]}$ 。最后, Luttbeg 等 ${ }^{\left[{ }^{[0]}\right.}$ 证明了外部生态条件对动物个性形成 的重要作用,动物个性的形成受到捕食风险和资源变化的强烈影响: 资源充足的环境有利于动物形成勇敢的 性格,而捕食压力强的环境则导致种群向谨慎的方向进化。

动物个性的形成与维持是一个复杂的问题, 相关的研究尚处于起步阶段, 研究者至今尚未找到动物个性 确切的形成机制。未来, 我们需要借助于动物行为学、分子遗传学、神经生物学和发育生物学等多学科交叉的 手段,才能进一步推动该领域的研究进展。此外,有效鉴定和识别动物个性差异背后的基因或基因组区域, 将 为研究分子水平的自然选择、基因之间与基因-环境间的相互作用关系以及基因表达如何塑造动物个性提供 更深层次的认识 ${ }^{[81]}$ 。

\section{5 展望}

综上所述, 个性广泛存在于动物个体中, 是调控动物个体行为、种群多度和分布、以及群落结构和功能的 
重要因子。当前国内关于动物个性的研究不多,但国际上对该领域的实验和理论研究已经相当深人。尽管如 此,动物个性的研究依然存在诸多局限性。

第一, 当前对动物个性的研究大多在实验室条件下进行, 缺乏在野外对动物个性的长期观测追踪工作。 在更复杂的自然环境中,动物个性是否可以长期维持? 动物个性的形成与维持如何受性别、发育阶段、种群密 度等因素的影响 ${ }^{[82]}$ ? 第二, 顶级捕食者个性在调控群落结构和动态中的作用尚未完全清楚 ${ }^{[59]}$ 。当前仅有的 几个关于动物个性对群落水平影响的研究, 大多集中于水生生态系统。尤其缺乏对陆地生态系统中大型顶级 捕食者个性的研究,一种或多种捕食者的个性差异,如何影响捕食者的集团内捕食(intraguild predation), 以及 捕食者对群落结构和功能的下行营养级联效应( top-down trophic cascade)? 由于大型顶级捕食者常常是一个 生态系统的关键种, 对其个性的深人研究有助于我们更好地理解生物群落的构建机制 ${ }^{[83]}$ 。第三, 缺乏对动物 个性时空变化格局的研究。当前研究者对单一生态系统中动物的个性开展了广泛研究, 但对于大尺度地理空 间以及长时间条件下, 动物个性的变化规律及其驱动因子尚缺乏深人了解。第四, 动物个性差异是生物多样 性的一个重要方面,但其重要性往往被忽视, 动物个性多样性化对种群、群落乃至整个生态系统在生态和进化 上的稳定性的影响有待深人探索。

最后, 环境变化对动物个性的影响正逐渐成为研究热点 ${ }^{[72-74]}$ 。随着人类活动和气候变化的加剧, 动物个 性对这些环境变化的响应及其机制逐渐成为动物行为和生态学研究的重要主题。一些研究开展了城市化对 动物个性影响的研究, 但对于其他环境变化因子, 如全球变暖、极端降雨、生境破碎化、物种人侵等对动物个性 的影响尚不多见。探索全球变化背景下动物个性的形成和维持机制, 以及个性类型对动物行为、种群多度和 分布以及种间关系等的反馈效应, 有助于我们更好地预测全球变化下生物多样性与群落构建的变化趋势, 从 而为自然生态系统的保护和管理提供科学依据。

\section{参考文献( References) :}

[ 1 ] Caspi A, Roberts B W, Shiner R L. Personality development: stability and change. Annual Review of Psychology, 2005, 56(1): 453-484.

[ 2 ] Gosling S D. From mice to men: what can we learn about personality from animal research? Psychological Bulletin, 2001, 127(1): 45-86.

[ 3 ] Dall S R X, Houston A I, McNamara J M. The behavioural ecology of personality: consistent individual differences from an adaptive perspective. Ecology Letters, 2004, 7(8): 734-739.

[ 4 ] Réale D, Reader S M, Sol D, McDougall P T, Dingemanse N J. Integrating animal temperament within ecology and evolution. Biological Reviews, $2007,82(2): 291-318$.

[ 5 ] Sih A, Bell A, Johnson J C. Behavioral syndromes: an ecological and evolutionary overview. Trends in Ecology \& Evolution, 2004, 19(7): 372-378.

[ 6 ] Koolhaas J M, Korte S M, De Boer S F, van der Vegt B J, van Reenen C G, Hopster H, de Jong I C, Ruis M A W, Blokhuis H J. Coping styles in animals: current status in behavior and stress-physiology. Neuroscience \& Biobehavioral Reviews, 1999, 23(7) : 925-935.

[ 7 ] 陈卓月, 赵大鹏, 李保国. 浅谈动物个性生物学研究. 生物学通报, 2014, 49(2): 4-6.

[ 8 ] Huntingford F A. The relationship between anti-predator behaviour and aggression among conspecifics in the three-spined stickleback, Gasterosteus Aculeatus. Animal Behaviour, 1976, 24(2) : 245-260.

[ 9 ] Sih A, Bell A M. Chapter 5 insights for behavioral ecology from behavioral syndromes. Advances in the Study of Behavior, 2008, 38( 8) : $227-281$.

[10] Wolf M, Weissing F J. Animal personalities: consequences for ecology and evolution. Trends in Ecology \& Evolution, 2012, $27(8)$ : 452-461.

[11] Zhong Z W, Li X F, Pearson D, Wang D L, Sanders D, Zhu Y, Wang L. Ecosystem engineering strengthens bottom-up and weakens top-down effects via trait-mediated indirect interactions. Proceedings of the Royal Society B: Biological Sciences, 2017, 284( 1863) : 20170475.

[12] Wang L, Wang D L, He Z B, Liu G F, Hodgkinson K C. Mechanisms linking plant species richness to foraging of a large herbivore. Journal of Applied Ecology, 2010, 47(4): 868-875.

[13] Sih A, Bell A M, Johnson J C, Ziemba R E. Behavioral syndromes: an integrative overview. The Quarterly Review of Biology, 2004, 79(3): 241277.

[14] Biro P A, Stamps J A. Are animal personality traits linked to life-history productivity? Trends in Ecology \& Evolution, 2008, 23(7) : 361-368.

[15] Jandt J M, Bengston S, Pinter-Wollman N, Pruitt J N, Raine N E, Dornhaus A, Sih A. Behavioural syndromes and social insects: personality at multiple levels. Biological Reviews, 2014, 89(1): 48-67.

[16] Carter A J, Feeney W E, Marshall H H, Cowlishaw G, Heinsohn R. Animal personality : what are behavioural ecologists measuring? Biological Reviews, 2013, 88(2): 465-475.

[17] 张嫱, 付世建, 夏继刚. 鱼类“个性”行为及其研究进展. 生态学杂志, 2017, 36(12) : 3623-3628.

[18] 邓可, 刘伟, 王德华. 小型哺乳动物种群调节中社会行为与亲缘结构联动机制研究进展. 生态学杂志, 2016, 35(3): 825-833.

[19] Lowe S E, Bradshaw J W S. Ontogeny of individuality in the domestic cat in the home environment. Animal Behaviour, 2001, 61(1) : 231-237. 
[20] Goldsmith H H, Buss A H, Plomin R, Rothbart M K, Thomas A, Chess S, Hinde R A, McCall R B. Roundtable: what is temperament? Four approaches. Child Development, 1987, 58(2) : 505-529.

[21] Box H O. Temperament and socially mediated learning among primates//Box H O, Gibson K R, eds. Mammalian Social Learning: Comparative and Ecological Perspectives. Cambridge: Cambridge University Press, 1999: 33-56.

[22] Budaev S V, Mikheev V N, Pavlov D S. Individual differences in behavior and mechanisms of ecological differentiation on the example of fish. Biology Bulletin Reviews, 2015, 5(5): 462-479.

[23] Sih A. Behavioral syndromes : a behavioral ecologist's view on the evolutionary and ecological implications of animal personalities//Weiss A, King J E, Murray L, eds. Personality and Temperament in Nonhuman Primates. New York: Springer, 2011: 313-336.

[24] Garamszegi L Z, Herczeg G. Behavioural syndromes, syndrome deviation and the within- and between-individual components of phenotypic correlations: when reality does not meet statistics. Behavioral Ecology and Sociobiology, 2012, 66( 12) : 1651-1658.

[25] Wilson D S. Adaptive individual differences within single populations. Philosophical Transactions of the Royal Society B: Biological Sciences, $1998,353(1366):$ 199-205.

[26] 周杨, 黎勇, 周密, 孟秀祥. 圈养马鹿的胆量个性及与鹿香分泌和繁殖成效的关系. 兽类学报, 2018, 38(4): 344-351.

[27] Dingemanse N J, Both C, Drent P J, Tinbergen J M. Fitness consequences of avian personalities in a fluctuating environment. Proceedings of the Royal Society B: Biological Sciences, 2004, 271(1541): 847-852.

[28] Riechert S E, Hedrick A V. A test for correlations among fitness-linked behavioural traits in the spider Agelenopsis aperta (Araneae, Agelenidae). Animal Behaviour, 1993, 46(4) : 669-675.

[29] Farwell M, McLaughlin R L. Alternative foraging tactics and risk taking in brook charr (Salvelinus fontinalis). Behavioral Ecology, 2009, 20( 5) : 913-921.

[30] Killen S S, Marras S, McKenzie D J. Fuel, fasting, fear: routine metabolic rate and food deprivation exert synergistic effects on risk-taking in individual juvenile European sea bass. Journal of Animal Ecology, 2011, 80(5): 1024-1033.

[31] Cote J, Dreiss A, Clobert J. Social personality trait and fitness. Proceedings of the Royal Society B: Biological Sciences, 2008, 275 (1653) : 28512858.

[32] Godin J G, Dugatkin L A. Female mating preference for bold males in the guppy, Poecilia reticulata. Proceedings of the National Academy of Sciences of the United States of America, 1996, 93(19) : 10262-10267.

[33] van Oers K, Drent P J, de Goede P, van Noordwijk A J. Realized heritability and repeatability of risk-taking behaviour in relation to avian personalities. Proceedings of the Royal Society B: Biological Sciences, 2004, 271 (1534) : 65-73.

[34] Coleman K, Wilson D S. Shyness and boldness in pumpkinseed sunfish: individual differences are context-specific. Animal Behaviour, 1998 , 56 (4) : 927-936.

[35] Malmkvist J, Hansen S W. The welfare of farmed mink (Mustela Vison) in relation to behavioural selection: a review. Animal Welfare, 2001, 10 (1) : 41-52.

[36] Jones R B. Experimental novelty and tonic immobility in chickens (Gallus domesticus). Behavioural Processes, 1984, 9(2/3) : 255-260.

[37] Archer J. Tests for emotionality in rats and mice: a review. Animal Behaviour, 1973, 21(2): 205-235.

[38] File S E, Wardill A G. Validity of head-dipping as a measure of exploration in a modified hole-board. Psychopharmacologia, 1975 , 44( 1) : 53-59.

[39] Wilson R C, Vacek T, Lanier D L, Dewsbury D A. Open-field behavior in muroid rodents. Behavioral Biology, 1976, 17(4) : 495-506.

[40] Faure J M, Mills A D. Improving the adaptability of animals by selection//Grandin T, Deesing M J, eds. Genetics and the Behavior of Domestic Animals. 2nd ed. Amsterdam: Academic Press, 2014: 291-316.

[41] Benus R F, Röndigs M. Patterns of maternal effort in mouse lines bidirectionally selected for aggression. Animal Behaviour, 1996,51 (1): 67-75.

[42] Richardson K M, Parlato E H, Walker L K, Parker K A, Ewen J G, Armstrong D P. Links between personality, early natal nutrition and survival of a threatened bird. Philosophical Transactions of the Royal Society B: Biological Sciences, 2019, 374( 1781$)$ : 20190373.

[43] Ballew N G, Mittelbach G G, Scribner K T. Fitness consequences of boldness in juvenile and adult largemouth bass. The American Naturalist, $2017,189(4): 396-406$.

[44] Stamps J A. Growth-mortality tradeoffs and 'personality traits' in animals. Ecology Letters, 2007, 10( 5) : 355-363.

[45] Werner E E, Anholt B R. Ecological consequences of the trade-off between growth and mortality rates mediated by foraging activity. The American Naturalist, 1993, 142(2): 242-272.

[46] Biro P A, Abrahams M V, Post J R, Parkinson E A. Behavioural trade-offs between growth and mortality explain evolution of submaximal growth rates. Journal of Animal Ecology, 2006, 75(5): 1165-1171.

[47] Schuett W, Tregenza T, Dall S R X. Sexual selection and animal personality. Biological Reviews, 2010, 85(2): 217-246.

[48] Firth J A, Cole E F, Ioannou C C, Quinn J L, Aplin L M, Culina A, McMahon K, Sheldon B C. Personality shapes pair bonding in a wild bird social system. Nature Ecology \& Evolution, 2018, 2(11): 1696-1699.

[49] Both C, Dingemanse N J, Drent P J, Tinbergen J M. Pairs of extreme avian personalities have highest reproductive success. Journal of Animal Ecology, 2005, 74(4): 667-674.

[50] Martin-Wintle M S, Shepherdson D, Zhang G Q, Huang Y, Luo B, Swaisgood R R. Do opposites attract? Effects of personality matching in breeding pairs of captive giant pandas on reproductive success. Biological Conservation, 2017, 207: 27-37.

[51] Bolnick D I, Amarasekare P, Araújo M S, Bürger R, Levine J M, Novak M, Rudolf V H W, Schreiber S J, Urban M C, Vasseur D A. Why intraspecific trait variation matters in community ecology. Trends in Ecology \& Evolution, 2011, 26( 4) : 183-192.

[52] Sih A, Cote J, Evans M, Fogarty S, Pruitt J. Ecological implications of behavioural syndromes. Ecology Letters, 2012, 15(3) : 278-289.

[53] Bell A M. Behavioural differences between individuals and two populations of stickleback (Gasterosteus aculeatus). Journal of Evolutionary Biology, $2005,18(2): 464-473$.

[54] Duckworth R A, Badyaev A V. Coupling of dispersal and aggression facilitates the rapid range expansion of a passerine bird. Proceedings of the 
National Academy of Sciences of the United States of America, 2007, 104(38): 15017-15022.

[55] Sih A, Kats L B, Maurer E F. Behavioural correlations across situations and the evolution of antipredator behaviour in a sunfish-salamander system. Animal Behaviour, 2003, 65(1): 29-44.

[56] Nagelkerken I, Munday P L. Animal behaviour shapes the ecological effects of ocean acidification and warming: moving from individual to community-level responses. Global Change Biology, 2016, 22(3) : 974-989.

[57] Peacor S D, Pangle K L, Schiesari L, Werner E E. Scaling-up anti-predator phenotypic responses of prey: impacts over multiple generations in a complex aquatic community. Proceedings of the Royal Society B: Biological Sciences, 2012, 279(1726) : 122-128.

[58] Post D M, Palkovacs E P, Schielke E G, Dodson S I. Intraspecific variation in a predator affects community structure and cascading trophic interactions. Ecology, 2008, 89(7) : 2019-2032.

[59] Start D, Gilbert B. Predator personality structures prey communities and trophic cascades. Ecology Letters, 2017, 20 (3) : 366-374.

[60] Bassar R D, Marshall M C, López-Sepulcre A, Zandonà E, Auer S K, Travis J, Pringle C M, Flecker A S, Thomas S A, Fraser D F, Reznick D N. Local adaptation in Trinidadian guppies alters ecosystem processes. Proceedings of the National Academy of Sciences of the United States of America, 2010, 107( 8) : 3616-3621.

[61] Krause J, James R, Croft D P. Personality in the context of social networks. Philosophical Transactions of the Royal Society B: Biological Sciences, 2010, 365(1560): 4099-4106.

[62] Pike T W, Samanta M, Lindström J, Royle N J. Behavioural phenotype affects social interactions in an animal network. Proceedings of the Royal Society B: Biological Sciences, 2008, 275(1650): 2515-2520.

[63] Barber I, Dingemanse N J. Parasitism and the evolutionary ecology of animal personality. Philosophical Transactions of the Royal Society B: Biological Sciences, 2010, 365(1560): 4077-4088.

[64] Boyer N, Réale D, Marmet J, Pisanu B, Chapuis J L. Personality, space use and tick load in an introduced population of Siberian chipmunks Tamias sibiricus. Journal of Animal Ecology, 2010, 79(3) : 538-547.

[65] Krause J, Croft D P, James R. Social network theory in the behavioural sciences: potential applications. Behavioral Ecology and Sociobiology, $2007,62(1): 15-27$.

[66] Michelena P, Jeanson R, Deneubourg J L, Sibbald A M. Personality and collective decision-making in foraging herbivores. Proceedings of the Royal Society B: Biological Sciences, 2010, 277(1684) : 1093-1099.

[67] Kurvers R H J M, van Oers K, Nolet B A, Jonker R M, van Wieren S E, Prins H H T, Ydenberg R C. Personality predicts the use of social information. Ecology Letters, 2010, 13(7): 829-837.

[68] Aplin L M, Farine D R, Morand-Ferron J, Cockburn A, Thornton A, Sheldon B C. Experimentally induced innovations lead to persistent culture via conformity in wild birds. Nature, 2015, 518(7540): 538-541.

[69] Chapple D G, Simmonds S M, Wong B B M. Can behavioral and personality traits influence the success of unintentional species introductions? Trends in Ecology \& Evolution, 2012, 27(1): 57-64.

[70] Holtmann B, Santos E S A, Lara C E, Nakagawa S. Personality-matching habitat choice, rather than behavioural plasticity, is a likely driver of a phenotype-environment covariance. Proceedings of the Royal Society B: Biological Sciences, 2017, 284( 1864 ) : 20170943.

[71] Samia D S M, Nakagawa S, Nomura F, Rangel T F, Blumstein D T. Increased tolerance to humans among disturbed wildlife. Nature Communications, 2015, 6(1): 8877 .

[72] Lapiedra O, Chejanovski Z, Kolbe J J. Urbanization and biological invasion shape animal personalities. Global Change Biology, 2017, 23(2): 592-603.

[73] Sol D, González-Lagos C, Moreira D, Maspons J, Lapiedra O. Urbanisation tolerance and the loss of avian diversity. Ecology Letters, 2014 , 17 ( 8) : 942-950.

[74] Miranda A C, Schielzeth H, Sonntag T, Partecke J. Urbanization and its effects on personality traits: a result of microevolution or phenotypic plasticity? Global Change Biology, 2013, 19(9) : 2634-2644.

[75] Wolf M, van Doorn G S, Leimar O, Weissing F J. Life-history trade-offs favour the evolution of animal personalities. Nature, 2007, 447( 7144) : 581-584.

[76] 程琪, 曲家鹏, 张贺, 张堰铭. 高原鼠兔个性特征的地理变异及其对环境的适应. 兽类学报, 2017, 37(2)：115-123.

[77] Réale D, Garant D, Humphries M M, Bergeron P, Careau V, Montiglio P O. Personality and the emergence of the pace-of-life syndrome concept at the population level. Philosophical Transactions of the Royal Society B: Biological Sciences, 2010, 365(1560): $4051-4063$.

[78] Koolhaas J M, de Boer S F, Coppens C M, Buwalda B. Neuroendocrinology of coping styles: towards understanding the biology of individual variation. Frontiers in Neuroendocrinology, 2010, 31(3) : 307-321.

[79] Coppens C M, de Boer S F, Koolhaas J M. Coping styles and behavioural flexibility: towards underlying mechanisms. Philosophical Transactions of the Royal Society B: Biological Sciences, 2010, 365(1560): 4021-4028.

[80] Luttbeg B, Sih A. Risk, resources and state-dependent adaptive behavioural syndromes. Philosophical Transactions of the Royal Society B: Biological Sciences, 2010, 365(1560) : 3977-3990.

[81] van Oers K, Mueller J C. Evolutionary genomics of animal personality. Philosophical Transactions of the Royal Society B: Biological Sciences, $2010,365(1560): 3991-4000$.

[82] Stamps J, Groothuis T G G. The development of animal personality: relevance, concepts and perspectives. Biological Reviews, 2010, 85(2) : 301-325.

[83 ] Lapiedra O, Schoener T W, Leal M, Losos J B, Kolbe J J. Predator-driven natural selection on risk-taking behavior in anole lizards. Science, $2018,360(6392): 1017-1020$. 